\title{
Study of the SIR Model and Improvement Based on Applications
}

\author{
Siyuan Zhang ${ }^{1, a}$ \\ ${ }^{1}$ North China Electric Power University(Baoding), Baoding 071000, China \\ a593736114@qq.com
}

Keywords: SIR model, differential equation model, Ebola Virus, numerical computation, treatment

\begin{abstract}
Based on the classical SIR model, we improve the differential equation modal and take the effect of drugs and vaccines into account where Ebola viruses appear. Despite of the improvement, differential equation model is still the principle part of our model. Since the spread of Ebola is concerned with the region, this model and the subsequent discussion are established on the basis of a relatively closed district.

We first define variables related to drugs and vaccines and improve the chance of rehhabilitation in SIR model with respect to the quantity of medicine. Besides, we add a fourth type of crowd, the dead people. Nevertheless, we still classify the dead into the total population in the district and consider the total population is still constant.

Numerical computation reveals tendency of the infective with different initial conditions, which is served as a reflection of the spread of Ebola. Based on the phase trajectory, we construct a relationship between the infective and the susceptible and find the threshold concerned with medicine. What's more, we analyze the sensitivity as well as the robustness and conclude that values of the parameters will change the result dramatically.
\end{abstract}

\section{Terminology and Definitions}

Susceptible: Healthy people,susceptible to the Ebola Virus Disease and can be infected with Ebola. Infective: Patients who have been infected with Ebola and are fighting with the disease.

Removed: People recovered from Ebola Virus Disease with immunity to Ebola or susceptible with immunity acquired from vaccines.

Dead: People died from Ebola and Ebola is the only cause of the unnatural deaths.

\section{Differential Equation Model Based on the SIR Model}

\section{Differential Equation Model}

Parameters required are listed below. In addition to parameters in the classical SIR model, we add several new variables and improve some concepts considering the drugs and vaccines, as well as the morality. The principle part of our model is still the differential equations, with the unknown concepts newly defined.

\section{Model parameters}

Parameters Meaning

\begin{tabular}{cc}
\hline $\mathrm{N}$ & Total population in the district with spread of Ebola \\
$\mathrm{s}(\mathrm{t})$ & The proportion of the susceptible in the total population $\mathrm{N}$ \\
$\mathrm{i}(\mathrm{t})$ & The proportion of the infective in the total population $\mathrm{N}$ \\
$\mathrm{r}(\mathrm{t})$ & The proportion of the removed in the total population $\mathrm{N}$ \\
$\mathrm{h}(\mathrm{t})$ & The proportion of the dead in the total population $\mathrm{N}$ \\
$\mathrm{c}$ & The average number of effective contacts per patient per day, \\
which is the daily contact rate
\end{tabular}

\section{Step 1.Speeds of the drugs and vaccines delivered to the hospital}

Before the duration of delivery of the drugs $\Delta t_{1}$ and that of the vaccines $\Delta t_{2}$, the speeds of them delivered to the hospital both equal zero. After their respective durations of delivery, however, 
drugs and vaccines arrive successively. Besides, the speed of the supplements equals to that of the production respectively. And we have assumed that the company produce drugs and vaccines at the maximum rate continuously, the speeds of production $V_{p}$ and $V_{y}$ are constants.

As a result, after $\Delta t_{1}$ and $\Delta t_{2}$, the speed of the drugs delivered to the hospital $V d$ and the speed of the vaccines delivered to the hospital $V_{v}$ will be equal to the constants $V_{p}$ and ${ }^{V y}$ respectively. Thus, $V_{d}$ and $V_{v}$ can be expressed in piecewise functions presented below

$$
\begin{aligned}
& v_{d}= \begin{cases}0, & \mathrm{t} \leq \Delta t 1 \\
v_{p}, & \mathrm{t}>\Delta t 1\end{cases} \\
& v_{v}= \begin{cases}0, & \mathrm{t} \leq \Delta t 2 \\
v y, & \mathrm{t}>\Delta t 2\end{cases}
\end{aligned}
$$

We need to notice that $\Delta t_{1}$ is proportional to ${ }^{l}$ and $\Delta t_{2}$ is proportional to ${ }^{l_{2}}$, which can be described in mathematical language as

$\Delta t_{1} \propto l_{1}$

$\Delta t_{2} \propto l_{2} . \quad l_{1}$ and $l_{2}$ are the reflections of the locations of delivery.

\section{Step 2.Concepts about various quantities of drugs}

Since the quantity of the medicine needed is identical for each patient at any moment, and the medicine taken before has no effect on this moment in our assumptions, the quantity of the drugs needed for total patients at time $t$ is proportional to the number of the infective, which is the proportion of the infective $\mathrm{i}(\mathrm{t})$ times the total population $\mathrm{N}$. And the number of the infective times the requirement of drugs of each patient q will result in the total quantity needed ${ }^{Q_{n}}$.Because of the fact that $\mathrm{i}(\mathrm{t})$ is a function with time as the independent variable, ${ }^{{ }^{n}}$ is time-dependent as well. Therefore, the quantity of the medicine needed at time $t$ can be presented as ${ }^{Q_{n}}(\mathrm{t})=\mathrm{qNi}(\mathrm{t})$.

The quantity of the drugs stocked in the hospital $Q_{s}$ is a time-dependent variable since it is related to the quantity of drugs distributed to the injective at time $t$ and the speed of drugs delivered to the hospital, therefore, it is more reasonable to express the quantity of the drugs stocked in the hospital as $Q_{s}(\mathrm{t})$. The derivative of $Q^{s}(\mathrm{t})$ represents the supplementary speed of the drugs stocked in the hospital, which can be described as

$$
\frac{d Q_{s}(t)}{d t}=-Q_{d}(t)+v_{d} \text {, in which } Q_{d}(\mathrm{t}) \text { represe }
$$

In the case of sufficient drugs stocked in the hospital, the quantity of drugs distributed to the injective equals the quantity of drugs needed for all the patients $Q^{{ }^{n}}(\mathrm{t})$.Nevertheless, in face of the scarcity of the drugs, the hospital is demanded to distribute the entire stock to the needed, that is to say, the drugs stocked in the hospital will all be distributed to the injective, and the quantity of drugs distributed to the injective equals the quantity of drugs stocked in the hospital $Q_{s}$ (t).Consequently, the quantity of drugs distributed to the injective $Q_{d}(\mathrm{t})$ can also be expressed in a piecewise function

$$
Q_{d}(\mathrm{t})= \begin{cases}Q_{s}(\mathrm{t}), & Q_{s}(\mathrm{t}) \leq Q_{n}(\mathrm{t}) \\ Q_{n}(\mathrm{t}), & Q_{s}(\mathrm{t})>Q_{n}(\mathrm{t})\end{cases}
$$


Nevertheless, we are able to change the form of the piecewise function to a min function or an absolute value function shown as

$$
\begin{aligned}
Q_{d}(\mathrm{t}) & =\min \left\{{ }^{Q_{s}}(\mathrm{t}),{ }^{Q_{n}}(\mathrm{t})\right\}, \\
& \quad Q_{d}(t)=\frac{Q_{s}(t)+Q_{n}(t)-\left|Q_{s}(t)-Q_{n}(t)\right|}{2} .
\end{aligned}
$$

The three time-dependent variables listed above reveal the quantities of the drugs for different applications and they are in close ties.

\section{Step 3.The chance of rehabilitation}

In our model, the chance of rehabilitation $f$ is related to $Q_{d}$ and $Q_{n}$ in a linear form $\mathrm{y}=\mathrm{ax}+\mathrm{b}$. Combining our assumptions, the chance of rehabilitation $f$ can be presented as

$$
f=\gamma Q_{d}(\mathrm{t}) / Q^{Q_{n}}(\mathrm{t})+\mathrm{c},
$$

in which c is the proportion of the cured in the total infective without drugs. What's more, it is obvious that $\gamma>0, \mathrm{c}>0$, since the chance of rehabilitation $f$ is in a positive correlation to $Q_{d}(\mathrm{t}) / Q_{n}$ $(\mathrm{t})$,and the proportion of the cured in the total infective without drugs c can't be negative. And the range of $f$ locates between 0 and 1 considering the morality.

\section{Step 4.Differential equations based on the SIR model}

Figure 1 reveals the transformation of the four kinds of people as well as the spread of ebola virus among the suseptible, the infective, the removed and the dead.

For the infective,each patient is able to infect $\lambda \mathrm{s}(\mathrm{t})$ healthy people effectively and make them suffer from Ebola Virus Disease,so $\lambda \mathrm{Ns}(\mathrm{t}) \mathrm{i}(\mathrm{t})$ of the susceptible will be infected with Ebola each day.Since the chance of rehabilitation is $f, f \mathrm{Ni}(\mathrm{t})$ of the infective recover from the Ebola Virus Disease and transform into theremoved.

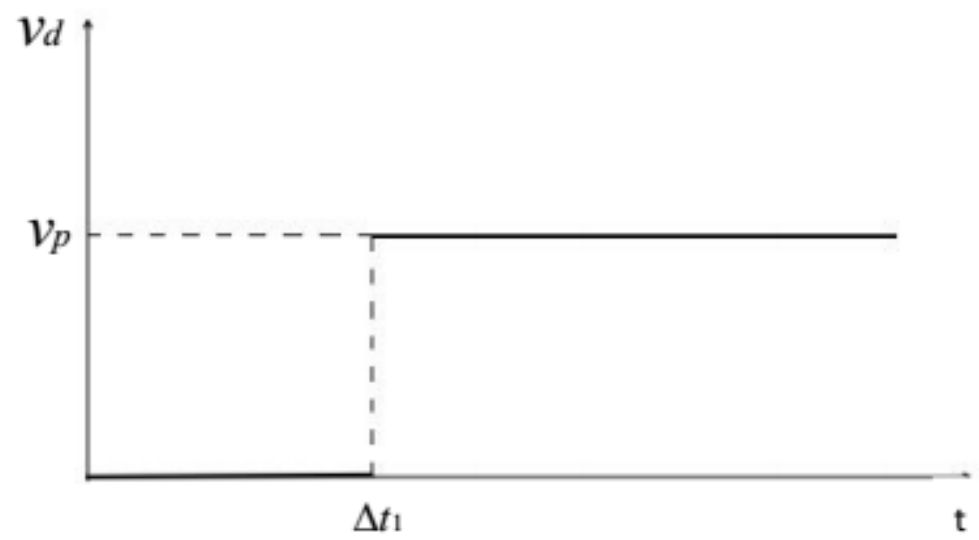

Transformation of the four kinds of people and the spread of ebolavirus

Considering the motality, we have assumed that the probability of death is in a first-order negative correlation with the intake of drugs per patient, so the probability of death is proportional to (1-f). Since $f=\gamma{ }^{Q_{d}}(\mathrm{t}) / Q^{Q_{n}}(\mathrm{t})+\mathrm{c}$, when there is not any drug distributed to the infective, $f=\mathrm{c}$. In this situation, $(1-f)=(1-c)$, while $(1-c) \eta$ is just the proportion of the dead in the total infective when there is no drugs. Thus, the number of patients died from Ebola equals $(1-f) \eta N i$. In general, considering the increased patients, the removed from Ebola as well as the motality, the differential equation with respect to the infective i(t) can be expressed as

$N \frac{d i}{d t}=\lambda N s i-f N i-(1-f) \eta N i$

After deformation, we get

$\frac{d i}{d t}=\lambda s i-f i-(1-f) \eta i$ 
For the susceptible, $\lambda \mathrm{s}(\mathrm{t})$ healthy people is infected to the Ebola Virus Disease effectively by a single patient at time $\mathrm{t}$, so $\lambda \mathrm{Ns}(\mathrm{t}) \mathrm{i}(\mathrm{t})$ of the susceptible will be infected with Ebola each day. Taking the vaccines into account, healthy people injected with the vaccines will obtain the immunity and become the removed. Since the speed of the vaccines produced $V_{y}$ and the speed of the vaccines delivered to the hospital $V_{v}$ are measured by the required quantity of vaccines for each healthy person, we could consider $V_{v}$ multiplied by the unit of time and the result is the susceptible vaccinated, the number of which equals $v_{v}$ in the magnitude. Besides, the susceptible vaccinated transforms into the removed. Therefore, considering the infected and vaccinated, the differential equation with respect to the susceptible $\mathrm{s}(\mathrm{t})$ can be expressed as

$$
\frac{\mathrm{ds}}{d t}=-\lambda s i-\frac{v_{v}}{N} \text {. }
$$

For the removed, $f \mathrm{Ni}(\mathrm{t})$ of the infective recover from the Ebola Virus Disease and transform into the removed under the condition of the chance of rehabilitation $f$. In addition, the susceptible vaccinated with the number of $V^{v}$ transforms into the removed.As a consequence,the differential equation with respect to the removed $\mathrm{r}(\mathrm{t})$ can be described as

$$
\frac{\mathrm{dr}}{d t}=f i+\frac{v_{v}}{N} \text {. }
$$

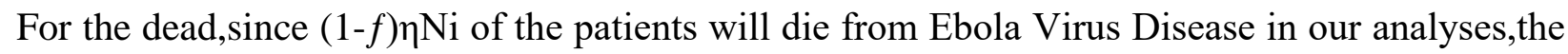
differential equation with respect to the dead $\mathrm{h}(\mathrm{t})$ can be expressed as

$$
\frac{d h}{d t}=(1-f) \eta i
$$

\section{Step 5.An essential constraint in accordance with our assumptions}

In our assumptions, we have supposed the total population to be divided into four sections:the susceptible,the infective,the removed and the dead.Consequently,the proportions of the foour parts add up to 1 compulsively, which is consistent with the assumption. And the constraint condition is $\mathrm{s}(\mathrm{t})+\mathrm{i}(\mathrm{t})+\mathrm{r}(\mathrm{t})+\mathrm{h}(\mathrm{t})=1$.

\section{Step 6.Initial conditions}

We integrate the initial conditions: $\mathrm{s}(0)=S_{0}, \mathrm{i}(0)=i_{0}, \mathrm{r}(0)=r_{0}, \mathrm{~h}(0)=h_{0}$. Besides, they satisfy the constraint as $S_{0}+i_{0}+r_{0}+h_{0}=1$.

\section{Validation of the model}

We collect the data from previous cases infected by the Zarie ebolavirus and draw the graphs of the susceptible, the infective, the removed and the dead in order to validate our model as well as reflect the effects of the medicine. Figure 6 shows the tendency of change of the four sections of people without medicine, while Figure 7 reveals that with the aid of medicine.

Statistics come from the official website of CDC(Centers for Disease Control and Prevention) and WHO(World Health Organization). Since the volume of data is tremendous, we will not list the data we used in drawing the graph.h(t) tends to be 1 in the situation without drugs, which is to say, nearly all people in the district will be infected with Ebola Virus Disease and die from the infectious disease in the end.The graph is consistent with the early society when Ebola was just discovered and medicine was not developed. The possibility of death in the second graph is much lower, reflecting drugs and vaccines have been invented and the treatment is improved, therefore, the spread of Ebola has been better controlled. 


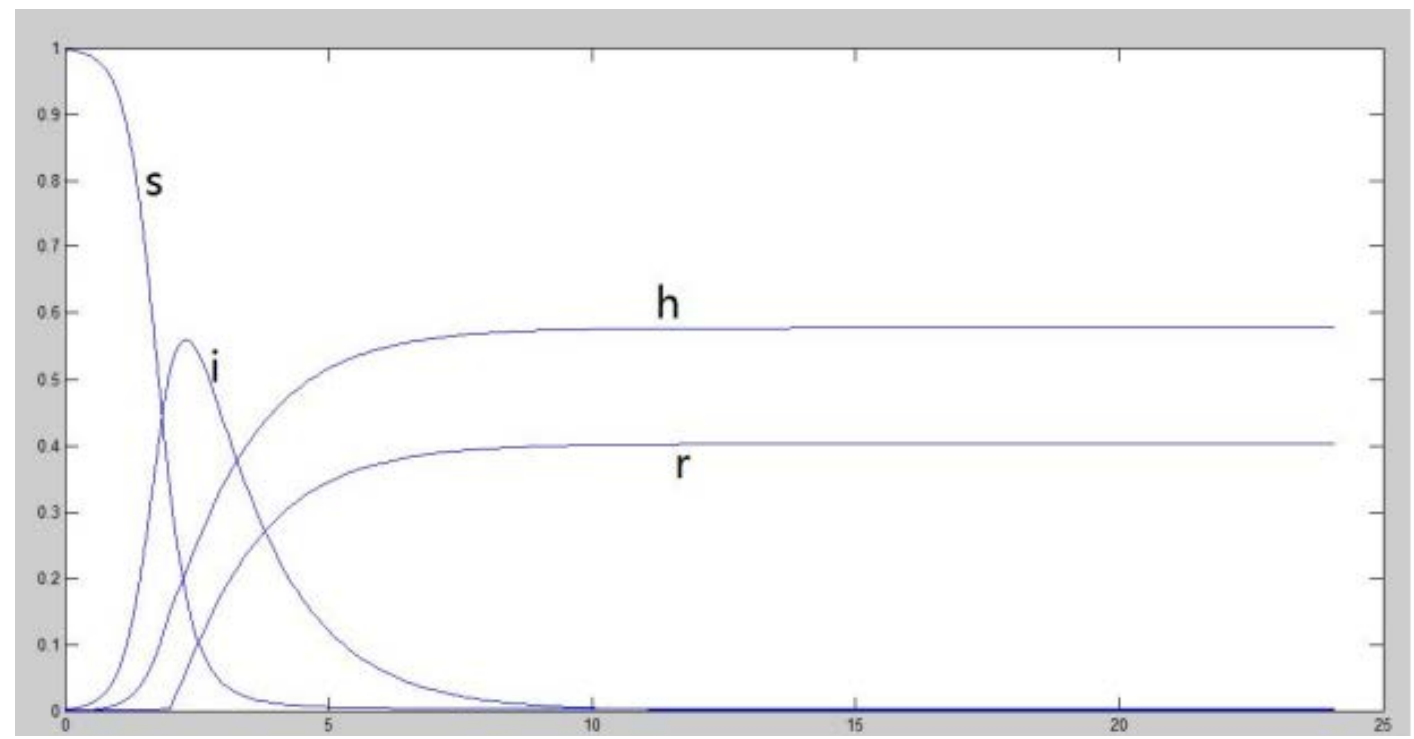

Tendency of the four sections of people with medicine

\section{Summary}

Ebola, an acute infectious disease, is required to be controlled as soon as possible. We are delighted for the development of the drugs and vaccines for the Ebola Virus Disease, and we establish a mathematical model based on a classical one to study and predict the spread of Ebola.

According to our results, the chance of rehabilitation closely concerned with medicine is a key to control the spread of Ebola. If patients infected with Ebola Virus Disease can be detected, isolated and treated as early as possible, the daily contact rate will be controlled and the cured patients will transform into the removed, therefore, there will be less infective to spread Ebola to the susceptible. Time is life, time lost on the transportation from factory to the hospital is a significant determination of whether the control of Ebola can be satisfied. Since the duration of delivery from drugs production company to the hospital will affect the control of the disease in a high level, hospit als would better prepare some stocks of Ebola medicine in prevention of the scarcity of drugs in face of the outbreak of Ebola.

What's more, vaccines will do help to control the spread of Ebola. Injection of the vaccine will transform a susceptible to the removed and reduce the number of people to be infected. Besides, it is likely to have some beneficial effect on the treatment of the patients and improve the chance of rehabilitation for the infective.

In addition, the medical and health level will affect the daily contact rate in a large way, it is of great importance to improve the environment as well as the therapeutic level in order to lower the infection.

\section{Reference}

[1] Cheng Y, Liu J, Li Y,et al.Ebola Virus Disease: virology, pathogenesis, therapy, andvaccines.Chin Sci Bull (Chin Ver), 2014, 59: 2889-2899,doi:10.1360/N972014-00884.

[2] LI Yu,Ren Xiang,Liu Di,Cheng Ying,Gao George Fu,Yu Hongjie.Ebola Virus Disease: epidemiology, ecology, diagnosis, treatment,and control. 2014, 32 (24), doi: 10.3981/j.issn.1000-7857.2014.24.001.

[3] Colebunders R, Borchert M. Ebola haemorrhagic fever-A review [J]. JInfect,2000,40(1):16-20.

[4] Le Guenno B, Formenty P, Wyers M, et al. Isolation and partial characterisation of a new strain of Ebola virus [J]. The Lancet,1995, 345:1271-1274. 